\title{
Recent researches in Wind Engineering at CRIACIV - Inter-University Research Center on Building Aerodynamics and Wind Engineering
}

\author{
Gianni BARTOLI * , Claudio MANNINI * , Antonino Maria MARRA* , \\ Tommaso MASSAI * , Luca PIGOLOTTI *
}

\section{Abstract}

The paper briefly summarizes some of the researches that have been running at CRIACIV in the last years, in order to give to the International Community a glimpse on the activities performed in the Wind Engineering Laboratory.

CRIACIV is an Inter-University Research Center focused on the field of Wind Engineering and Building Aerodynamics, grouping eight different Italian Universities. The Wind Engineering Laboratory is located in Prato, close to Florence, and it is equipped with a Boundary Layer Wind Tunnel. The Wind Tunnel test section is $2.42 \times 1.60 \mathrm{~m}$ (width $\times$ height), and a flow speed up to $30 \mathrm{~m} / \mathrm{s}$ can be reached with a longitudinal free stream turbulence level of about $0.7 \%$.
\end{abstract}

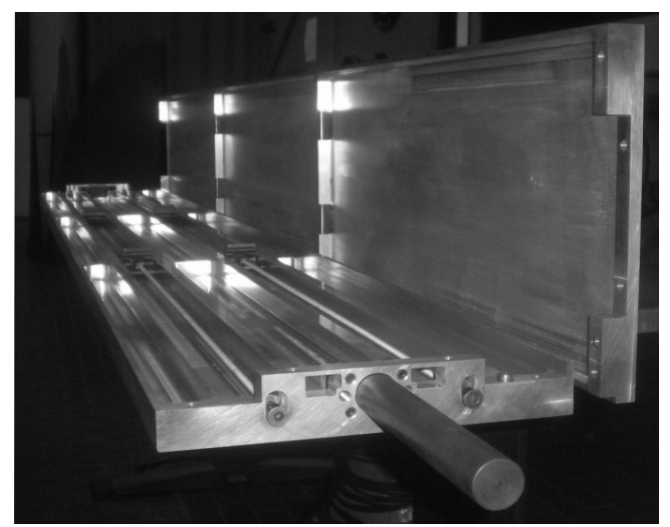

Among several different activities and ongoing researches at CRIACIV, some of them have been selected and reported in the paper. Those can be considered as the most interesting ones and a cooperative research at an International level could be envisaged.

Refined measurement on a sectional model for BARC investigations

During the 6th International Symposium on Bluff Body Aerodynamics and Applications (held in 2008 in Milan, Italy), a Benchmark on the Aerodynamics of a Rectangular 5:1 Cylinder was started (Bartoli et al. (2009)). By numerical and experimental approaches, BARC addresses the high Reynolds number, external,

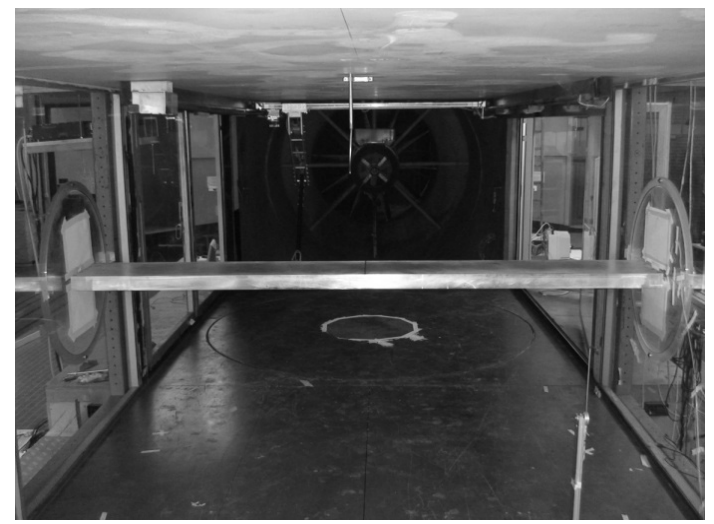

Figure 1. Left: view of the BARC model (open); right: view of the model in the CRIACIV wind tunnel.

* Inter-University Research Center on Building Aerodynamics and Wind Engineering / Department of Civil and Environmental Engineering, University of Florence, Italy 

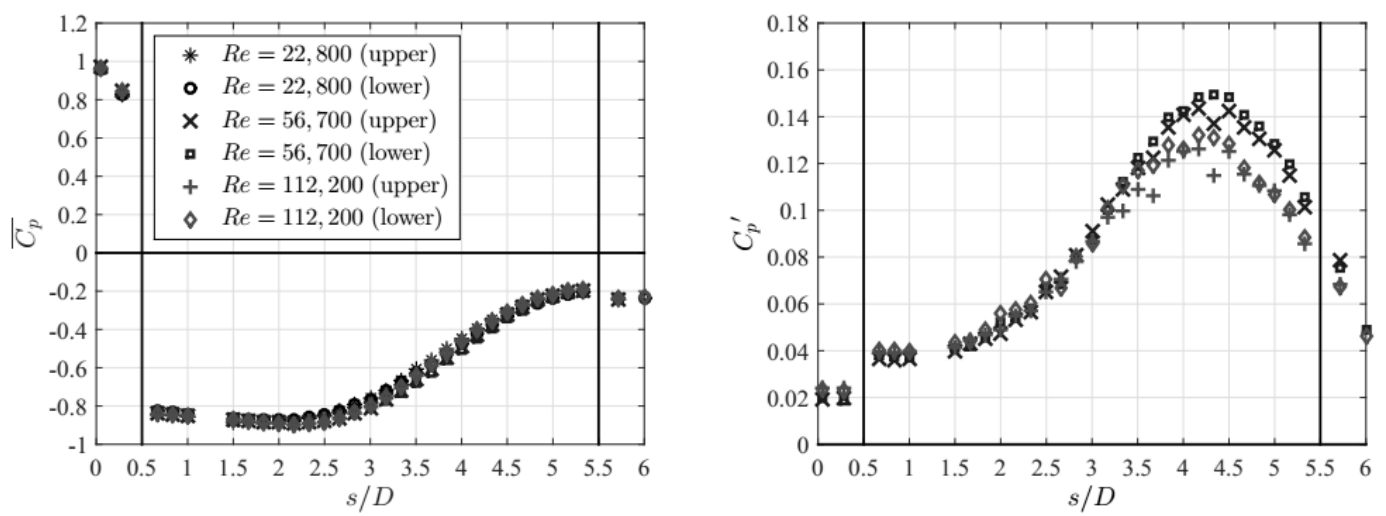

Figure 2. Mean and standard deviation of pressure coefficients in smooth flow for zero-degree angle of attack. $\mathrm{s} / \mathrm{D}$ is the nondimensional abscissa along the model section, $\mathrm{s} / \mathrm{D}=0$ and $\mathrm{s} / \mathrm{D}=6$ denoting respectively the windwardand leeward-face midpoint of the model (Mannini et al. 2016d).

unsteady flow around a stationary, sharp-edged rectangular cylinder, and the associated aerodynamic actions (Bartoli et al., 2008). The breadth (B) to depth (D) ratio was set equal to 5 as this side ratio is particularly interesting for some peculiar characteristics of the flow field close to the body (such as intermittent reattachment close to the trailing edges and more irregular vortex shedding mechanism as compared to similar side ratios).

Given the expected high sensitivity of the aerodynamic behavior of the rectangular 5:1 cylinder to several experimental conditions, a refined sectional model (Figure 1) has been designed with prismatic shape, sharp edges, smooth surfaces, absence of screws, etc. (as reported in Bartoli et al. (2011)). The accuracy of the model manufacturing allows to measuring more reliable experimental data to be also used as a reference to validate the results of Computational Fluid Dynamics simulations, where ideal geometric boundaries are usually assumed. Figure 2 shows some results of the experimental campaign recently conducted and reported in Mannini et al. (2016d) highlighting the good quality of the model as it can be appreciated by observing the symmetry of the pressure distributions along the two long faces. Extensive studies have also been carrying out in grid-generated turbulent flows.

The model could also be used as a "benchmark" itself among other laboratories, which could test it in similar flow conditions in order to cross check results from different Boundary Layer Wind Tunnels.

\section{Interference of VIV and galloping or low-speed galloping}

In the last years, a deep experimental campaign has been devoted to the study of the interaction between vortex-induced vibration (VIV) and galloping, also called low-speed galloping to take evidence of the fact that the galloping branch starts at the critical velocity of Kármán vortex resonance instead of that predicted by the quasi-steady theory. Even if the phenomenon has been addressed since several years ago (relevant references can be found in the outstanding works by Parkinson \& Brooks (1961) and Parkinson \& Smith (1964)), after an experimental campaign on a test case several aspects have been deepened and investigated, as illustrated in the recent review reported in Mannini et al. (2014).

At CRIACIV, experimental and theoretical works on this topic are developing. Since 2012, several wind tunnel campaigns have been carried out (Mannini et al., 2014, 2015, 2016a) on a rectangular 3:2 cylinder with the smallest face perpendicular to the oncoming flow. Recently, results reported in Mannini et al. (2016b, 2016c) helped understanding the dynamics of the interaction between the two excitation mechanisms and clearly highlighted the transition through four regimes 
of VIV-galloping interference. It was found that a high value of the mass-damping parameter is required to completely decouple the ranges of excitation due to vortex-induced vibration and galloping and for the quasi-steady theory to correctly predict the galloping critical wind speed (Figure 3). This conclusion is also relevant from the engineering point of view, as it means that structures and structural elements with ordinary mass-damping properties can exhibit sustained vibrations where they are not predicted by classical theories of vortex-induced vibration and galloping (see e.g. Mannini et al., 2016a).

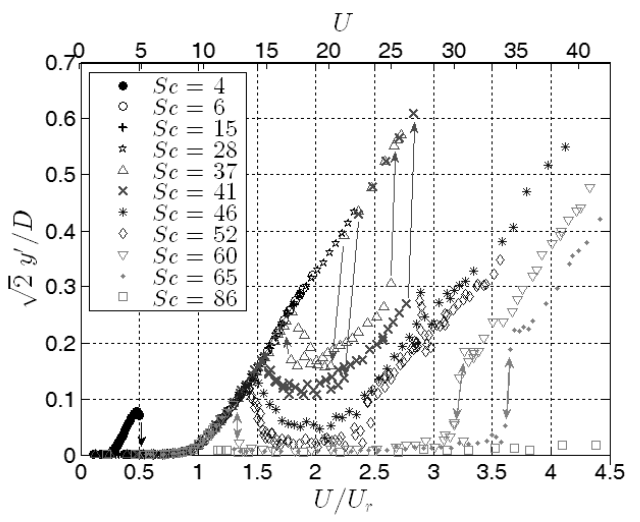

Figure 3. Summary of the amplitude-velocity curves for various Scruton numbers. $U$ and $U_{r}$ denote respectively the reduced flow speed and the vortex-resonance reduced flow speeds.

\section{Energy harvesting}

In the last years, a growing attention has been worldwide devoted to investigate the possibility of exploiting different fluid-structure interaction mechanisms capable of extracting energy from wind or water flows, by means of flow-induced vibrations of elastic systems. The following kinetic energy can be converted into other usable forms, among which electricity production is obtained by means of specific conversion apparatus based on vibrational electromagnetic transducers (e.g. Roundy, 2005). These recent energy harvesting systems usually show more flexibility of design and application (especially devoted to low-power installations) and promising performances, although their technology readiness level is still evolving.

The ongoing research at CRIACIV is focused on the study of power generators based on two-degree-of-freedom (2-DoF) classical flutter. Sectional models performing heaving and pitching oscillations are investigated through wind tunnel tests. Elongated rectangular cross sections with width-to-depth ratio of $25: 1$ are considered, with the smallest dimension facing the flow. A specific aeroelastic setup, with linear mechanical properties in the tested range of oscillations (see next section and Figure 4), has been developed to observe large motion amplitude and to set parametric investigations. It allows easily modifying some of the governing parameters of the flutter problem, in order to look for an optimal configuration for energy harvesting purposes. In these recent experimental campaigns, the energy extraction is simulated via additional damping in the heaving DoF (e.g. Karami \& Inman, 2011). Systematic parametric linear analyses are also implemented to find optimal configurations in terms of as low as possible critical reduced velocity.

As a general result, all tested configurations exhibit a dynamic instability with sub-critical bifurcation. Limit cycle oscillations with stable amplitudes are reached at the steady-state regime. Concerning energy harvesting purposes, a significant reduction of the critical wind speed is achieved by moving the mass center slightly downstream of the elastic axis (Pigolotti et al., 2016).

Flutter-based generators should also be designed with as low as possible inertia with respect to the moved flow. Although the performance factor is proportional to this mass-ratio parameter, the critical reduced velocity is intrinsically affected as well, as confirmed by the linear analysis, and the global effect is positive for energy generation. Finally, it is worth highlighting the importance of the sub-critical stable branch that represents an extension of the operative range in which the system can produce energy, if spontaneously reached from higher amplitudes or externally triggered.

\section{A new two-degree-of-freedom setup}

The usual 2-DoF aeroelastic setups adopted in wind tunnel tests rely on a coil-spring suspension, where the 

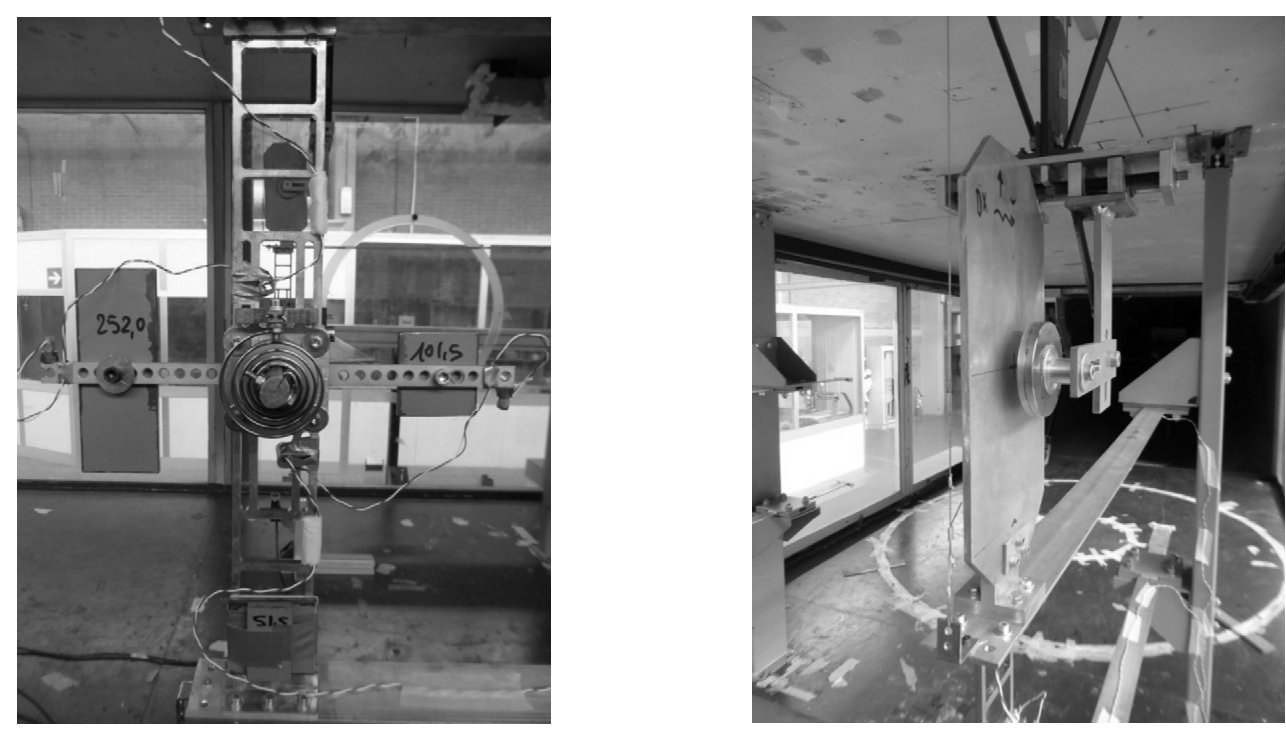

Figure 4. The new 2-DOF setup for bodies undergoing large oscillations.

Left: view of the clock-spring used to give stiffness in the pitching degree of freedom; right: cantilevered suspending beams for the heaving degree of freedom, with the magnetic dampers installed.

pitching stiffness can be modified by varying the distance between the pairs of springs. This typology is mainly adopted for small oscillation amplitudes because of the intrinsic geometric nonlinearity for large rotational amplitudes. In the case of large motion, setups with a suspension obtained with blade-springs for the heaving DoF and peculiar expedients for the pitching DoF, such as clock springs, are preferred.

A large-amplitude, 2-DoF blade-spring setup (Figure 4) has been developed at CRIACIV, essentially to observe post-critical regimes in flutter or galloping of sectional models. Two pairs of cantilevered flexible beams, each pair connected at the free ends by a Vieredeel girder to behave like a "shear-type" frame (i.e. allowing only transverse/vertical displacements), are combined with two pairs of coil springs to provide the heaving stiffness; a double ball-bearing system and two clock-springs are employed to decouple the two DoFs and to give stiffness to the torsional degree of freedom. The suspension is placed inside the test section and therefore is sheltered from the flow through streamlined screens. The mechanical properties are linear in the tested range of amplitudes, that is $\pm 100 \mathrm{~mm}$ of heaving and $\pm 90^{\circ}$ of pitching. Moreover, the damping coefficients resulted very low (about $0.05 \%$ in heaving and $1 \%$ in pitching); the heaving damping can be increased through eddy-current dampers. The frequency of heaving oscillation can be varied by modifying the free-length of the beams or the coil stiffness, while the pitching frequency is defined by the clock-spring stiffness.

\section{References}

Bartoli, G., Bruno, L., Buresti, G., Ricciardelli, F., Salvetti, M.V., Zasso, A., BARC overview document. $<$ http://www.aniv-iawe.org/barc>, 2008.

Bartoli, G., Bruno, L., Buresti, G., Ricciardelli, F., Salvetti, M.V., Zasso, A., BARC: a benchmark on the aerodynamics of a rectangular 5:1 cylinder, Journal of Fluids and Structures 25, 586, 2009.

Bartoli, G., Borsani, A., Mannini, C., Marra, A.M., Procino, L., Ricciardelli, F., Wind tunnel study on the aerodynamics of a 5:1 rectangular cylinder in smooth flow, Proceedings of the 13th International Conference on Wind Engineering, Amsterdam, The Netherlands, July 10-15, 2011.

Karami, M., Inman, D., Equivalent damping and frequency change for linear and nonlinear hybrid vibrational energy harvesting systems, Journal of Sound and Vibration 330 (23), 5583-5597, 2011. 
Mannini, C., Marra, A.M., Bartoli, G., VIV-galloping instability of rectangular cylinders: Review and new experiments, Journal of Wind Engineering and Industrial Aerodynamics, 132, 109-124, 2014.

Mannini, C., Marra, A.M., Bartoli, G., Experimental investigation on VIV-galloping interaction of a rectangular 3:2 cylinder, Meccanica 50 (3), 841-853, 2015.

Mannini, C., Belloli, M., Marra, A.M., Bayati, I., Giappino, S., Robustelli, F., Bartoli, G., Aeroelastic stability of two lon-span arch structures: A collaborative experience in two wind tunnel facilities, Engineering Structures 119, 252-263, 2016a.

Mannini, C., Marra, A.M., Massai, T., Bartoli, G., Interference of vortex-induced vibration and transverse galloping for a rectangular cylinder, Journal of Fluids and Structures 66, 403-423, 2016 b.

Mannini, C., Marra, A.M., Massai, T., Bartoli, G., Low-speed galloping for rectangular cylinders with side ratios larger than unity, 1st International Symposium on Flutter and its Application (ISFA2016),
Tokyo, Japan, May 15-17, $2016 \mathrm{c}$.

Mannini, C., Marra, A.M., Pigolotti, L., Bartoli, G., The effects of free-stream turbulence and angle of attack on the aerodynamics of a cylinder with rectangular 5:1 cross section, submitted to Journal of Wind Engineering and Industrial Aerodynamics, 2016d.

Parkinson, G.V., Brooks, N.P.H., On the aeroelastic instability of bluff cylinders, Journal of Applied Mechanics 28 (2), 252-258, 1961.

Parkinson, G.V., Smith, J.D., The square prism as an aeroelastic non-linear oscillator, Quarterly Journal of Mechanics and Applied Mathematics 17 (2), 225-239, 1964.

Pigolotti, L., Mannini, C., Bartoli, G., Effects of mass unbalance and high levels of external damping on the post-critical flutter of a flat plate, 1st International Symposium on Flutter and its Application (ISFA2016), Tokyo, Japan, May 15-17, 2016.

Roundy, S., On the effectiveness of vibration-based energy harvesting, Journal of Intelligent Material Systems and Structures 16 (10), 809-823, 2005. 